\title{
Derrame pericárdico maciço como manifestação inicial do hipotireoidismo
}

\author{
Massive pericardial effusion as initial manifestation of hypothyroidism
}

Ana Cláudia Pelegrinelli Thirone', Rafael Vinícius Danieli', Vanessa Marajó Fernandes Corrêa Ribeiro'

\section{SUMÁRIO}

Este estudo visa relatar um caso raro de derrame pericárdico maciço como manifestação inicial do hipotireoidismo. Paciente de 21 anos, feminina, previamente hígida, iniciou quadro súbito de dispneia ao repouso e edema de membros inferiores. Após internação, exames laboratoriais de rotina evidenciaram hipotireoidismo (TSH 146,14 mUI/L). Também foi realizado ecocardiograma, o qual mostrou derrame pericárdico importante. Instituiu-se terapêutica com levotiroxina, obtendo-se melhora do quadro sem necessidade de pericardiocentese. Após a alta hospitalar, a paciente foi acompanhada por 1 ano, com remissão total da dispneia e do edema. No entanto, iniciou com sintomatologia típica de hipotireoidismo e permaneceu com astenia, dislipidemia, ganho de peso e discreto derrame pericárdico ao final de 1 ano, mesmo com a otimização da dose de levotiroxina. Destaca-se, com esse caso, a necessidade de investigação precoce do hipotireoidismo em pacientes com derrame pericárdico. Arq Bras Endocrinol Metab. 2012;56(6):...

\section{SUMMARY}

The aim of this study is to report a rare case of massive pericardial effusion as initial manifestation of hypothyroidism. A previously healthy 21-year-old female patient suddenly began presenting dyspnea at rest and lower limb edema. Routine laboratory tests performed at admission showed hypothyroidism (TSH $146.14 \mathrm{mUI} / \mathrm{L}$ ) and echocardiography showed significant pericardial effusion. Therapy was instituted with levothyroxine, resulting in clinical improvement without pericardiocentesis. The patient was followed up for 1 year, with total remission of dyspnea and edema. However, she developed typical symptoms of hypothyroidism, and remained with asthenia, dyslipidemia, weight gain, and mild pericardial effusion at the end of one year, even with the optimization of the levothyroxine dose. This case highlights the need for early investigation of hypothyroidism in patients with pericardial effusion. Arq Bras Endocrinol Metab. 2012;56(6):383-7
'Departamento de Endocrinologia e Metabologia, Hospital Universitário, Universidade de Uberaba (Uniube), Uberaba, MG, Brasil

Correspondência para: Ana Cláudia Pelegrinelli Thirone Av. Santos Dumont, 1580 38050-400 - Uberaba, MG, Brasil acpthirone@yahoo.com.br

Recebido em 8/Dez/2011 Aceito em 22/Jul/2012

\section{INTRODUÇÃO}

$\mathrm{O}$ hipotireoidismo é uma doença sistêmica que geralmente se apresenta por meio de uma ampla sintomatologia, como fadiga, sonolência, perda de memória, ganho de peso, intolerância ao frio, entre outros, podendo até mesmo levar a comprometimento cardíaco (1). Uma das manifestações cardíacas mais comumente associadas a essa entidade é o derrame pericárdico, cuja incidência sofreu grande mudança, passando de $30 \%$ a $80 \%$ no passado, quando o diagnóstico era tardio (1-4), para $3 \%$ a $5 \%$ na atualidade
(3-6), haja vista a tendência ao diagnóstico precoce do hipotireoidismo (5). Como a instalação do derrame pericárdico normalmente é insidiosa e o saco pericárdico é distensível, o derrame raramente adquire volume significativo de forma a ocasionar sintomas $(1,7)$, de modo que a ocorrência de derrame pericárdico maciço torna-se um evento ainda mais inesperado, principalmente como manifestação inicial do hipotireoidismo. O presente trabalho visa relatar um caso raro de derrame pericárdico maciço como manifestação inicial do hipotireoidismo, com seguimento por 1 ano. 


\section{RELATO DO CASO}

Paciente de 21 anos, sexo feminino, deu entrada no Departamento de Cardiologia do Hospital Santa Catarina, no dia 16/9/2010, com quadro súbito de dispneia ao repouso e edema de membros inferiores. Negava quaisquer outros sintomas, inclusive os típicos de hipotireoidismo, como astenia, intolerância ao frio, ganho de peso, disfunção cognitiva, constipação, hiporexia, pele seca, rouquidão, entre outros (1). Negava, ainda, doenças preexistentes e referia uso apenas de anticoncepcional oral $(0,030 \mathrm{mg}$ de etinilestradiol e 0,075 $\mathrm{mg}$ de gestodeno). Ao exame físico, apresentava-se em regular estado geral, fácies atípica, afebril, visivelmente dispneica e com palidez cutâneo-mucosa. Murmúrio vesicular preservado sem ruídos adventícios e frequência respiratória de 28 incursões respiratórias por minuto. Bulhas rítmicas normofonéticas a dois tempos, sem sopros, com frequência cardíaca de 72 batimentos por minuto e pressão arterial de 110 x $70 \mathrm{mmHg}$. Abdome inocente. Presença de edema mole, simétrico, indolor e cacifo positivo em membros inferiores.

Com esse quadro, a paciente foi encaminhada para internação, sendo solicitados exames laboratoriais de rotina, eletrocardiograma e ecocardiograma, com os seguintes resultados: hemograma com hemácias $3,18 \mathrm{x}$ $10^{6} / \mathrm{mL}(4,0-5,2)$, hematócrito $28 \%(37,0-48,0)$, hemoglobina 9,8 g/dL (12,0-15,0), leucócitos 6.370/ $\mathrm{mL}(4.000-10.000)$ e plaquetas $238 \times 10^{3} / \mathrm{mL}(150-$ 400); glicemia de jejum $77 \mathrm{mg} / \mathrm{dL}$ (70-99); ureia 32 $\mathrm{mg} / \mathrm{dL}$ (15-43); creatinina $1,55 \mathrm{mg} / \mathrm{dL}(0,7-1,3)$; TSH 146,14 mUI/L (0,4-4,0); eletrocardiograma evidenciando ritmo sinusal, FC: 78 batimentos por minuto, complexos de baixa voltagem, ausência de bloqueios e sinais de isquemia; ecocardiograma (Figura 1) que mostrava ritmo cardíaco regular, dimensões e espessuras das paredes ventriculares e atriais sem alterações, ventrículos com função contrátil e diastólica normais, fração de ejeção de $80 \%$, válvulas mitral, tricúspide, aórtica e pulmonar com aspectos morfodinâmicos normais, aorta ascendente sem alterações, ausência de sinais de hipertensão arterial pulmonar, mas com presença de derrame pericárdico importante sem sinais de restrição. Diante desses resultados, do quadro clínico e da idade da paciente, foi aventada a hipótese de derrame pericárdico maciço secundário ao hipotireoidismo.

Destaca-se que, a despeito do quadro dispneico e do derrame pericárdico da paciente, ela não apresentava os sinais clássicos de tamponamento cardíaco: hipotensão arterial, abafamento de bulhas e turgência jugular.

Diante do quadro, foi iniciada terapêutica conservadora com levotiroxina $50 \mathrm{mcg}$ pela manhã, por 15 dias, e, após esse período, aumentada a dose para 100 mcg ao dia.

Após quatro dias de internação, a paciente permanecia estável com melhora parcial da dispneia. Recebeu alta hospitalar e foi encaminhada ao ambulatório de endocrinologia.

Em dois meses, a paciente compareceu à primeira consulta, no dia 4/11/2010, queixando-se de astenia, diminuição da acuidade visual, queda de cabelos, cervicalgia, lombalgia, dor intensa em membros inferiores, irregularidade menstrual e constipação intestinal. Negava dispneia e edema. Apesar do surgimento de sintomatologia de hipotireoidismo, a paciente relatava ter permanecido em tratamento regular com a medicação prescrita. Ao exame físico: bom estado geral,

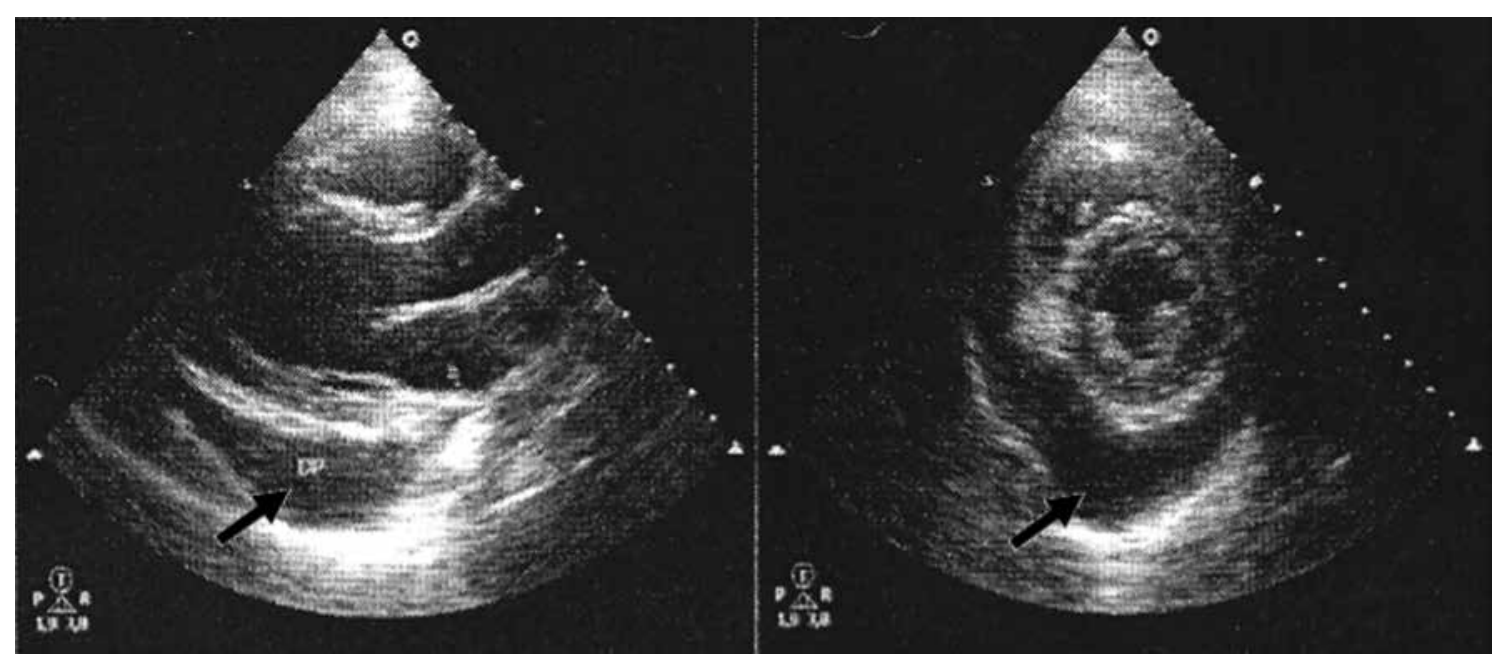

Figura 1. Ecocardiograma de 17/9/2010 evidenciando derrame pericárdico importante (setas). 
mucosas coradas, hidratadas, anictéricas e acianóticas, afebril, tireoide de tamanho e consistência normais à palpação, peso de $62,5 \mathrm{~kg}$, estatura de $1,67 \mathrm{~m}$ e índice de massa corporal (IMC) de $22,4 \mathrm{~kg} / \mathrm{m}^{2}$. Pulmões limpos. Ritmo cardíaco regular, normofonético, sem sopros, frequência cardíaca de 76 batimentos por minuto e pressão arterial de 110 x $70 \mathrm{mmHg}$. Abdome sem alterações. Membros inferiores sem edema. Foi mantida a dosagem de levotiroxina e solicitados novos exames.

No retorno, dia 25/11/2010, relatou melhora do padrão menstrual, assim como do hábito intestinal. Porém, houve aumento no peso de $2,5 \mathrm{~kg}$. Resultados de exames: hemograma com hemácias 3,6 x 10\% $\mathrm{mL}(4,0-5,2)$, hematócrito $32,5 \%(37,0-48,0)$, hemoglobina $10,7 \mathrm{~g} / \mathrm{dL}(12,0-15,0)$, leucócitos $4.900 / \mathrm{mL}$ (4.000-10.000) e plaquetas $219 \times 10^{3} / \mathrm{mL}(150-300)$; glicemia $88 \mathrm{mg} / \mathrm{dL}$ (70-99); ureia $34 \mathrm{mg} / \mathrm{dL}$ (15-43); creatinina $0,9 \mathrm{mg} / \mathrm{dL}(0,7-1,3)$; TSH $2,5 \mathrm{l} \mathrm{mUI} / \mathrm{L}(0,4-$ $4,0)$ e T4 livre $1,76 \mathrm{ng} / \mathrm{dL}(0,7-1,8)$; anti-TPO 412,3 $\mathrm{UI} / \mathrm{mL}(<35)$; perfil lipídico com colesterol total 214 $\mathrm{mg} / \mathrm{dL}(<200)$, HDL colesterol $72 \mathrm{mg} / \mathrm{dL}(>40)$, LDL colesterol $132 \mathrm{mg} / \mathrm{dL}(<130)$ e triglicérides 38 $\mathrm{mg} / \mathrm{dL}(<150)$. A dosagem de levotiroxina foi mantida e novos exames foram solicitados. Para redução do peso e correção da dislipidemia, foram orientadas mudanças comportamentais e atividade física.

Após 11 dias, a paciente compareceu à consulta com queixa de dispneia e precordialgia. Trouxe resultado do ecocardiograma de 3/12/2010 (Figura 2) que mostrava discreto derrame pericárdico associado a espessamento de folhetos. A paciente foi encaminhada para cardiologia e foram solicitados novos exames para diagnóstico diferencial, bem como para excluir outras doenças associadas, tendo em vista a presença de ma- nifestações atípicas, as quais, a princípio, não poderiam ser atribuídas apenas ao hipotireoidismo.

Em 27/1/2011, retornou referindo remissão total da dispneia e da precordialgia. Trouxe resultado de exames: hemácias $4,35 \times 10^{6} / \mathrm{mL}(4,0-5,2)$, hematócrito $37,7 \%(37,0-48,0)$, hemoglobina $12,6 \mathrm{~g} / \mathrm{dL}(12,0-$ $15,0)$, leucograma e plaquetograma sem alterações; ureia $31 \mathrm{mg} / \mathrm{dL}(15-43)$; creatinina $0,8 \mathrm{mg} / \mathrm{dL}(0,7$ $1,3)$; fator antinuclear não reagente; fator reumatoide 1,9 UI/mL (0-20); anticorpo anti-Sm não reagente; anticorpo anti-SSa/Ro não reagente; pesquisa de células LE no sangue negativa; PCR $3,7 \mathrm{mg} / \mathrm{L}(<5,0)$; TSH $8,11 \mathrm{mUI} / \mathrm{L}(0,4-4,0)$ e T4 livre $1,3 \mathrm{ng} / \mathrm{dL}$ (0,7-1,8). Em razão do aumento do TSH, a dosagem de levotiroxina foi ajustada para $112 \mathrm{mcg} / \mathrm{dL}$.

Em 18/3/2011, a paciente permanecia com queixa de dor intensa em membros inferiores. Trouxe TSH $3,47 \mathrm{mUI} / \mathrm{L}(0,4-4,0)$ e T4 livre $1,2 \mathrm{ng} / \mathrm{dL}(0,7-1,8)$. Como a paciente apresentava TSH acima da meta proposta pela Diretriz da Associação Americana de Endocrinologistas Clínicos para a Avaliação e Tratamento do Hipertireoidismo e Hipotireoidismo (8), que preconiza que o TSH deve ser mantido entre 0,3 e $3,0 \mathrm{mUI} / \mathrm{L}$, optou-se por aumentar a dosagem de levotiroxina para $125 \mathrm{mcg} /$ dia.

No dia 13/6/2011, a paciente afirmava estar assintomática. Entretanto, apresentou ganho ponderal, estando com peso de $66,5 \mathrm{~kg}$. Trouxe TSH 2,25 mUI/L $(0,4-4,0), \mathrm{T} 4$ livre $1,54 \mathrm{ng} / \mathrm{dL}(0,7-1,8)$, colesterol total $250 \mathrm{mg} / \mathrm{dL}(<200)$ e triglicérides $109 \mathrm{mg} /$ $\mathrm{dL}(<150)$ de $2 / 6 / 2011$; novo ecocardiograma de $17 / 3 / 2011$ evidenciava um derrame pericárdico laminar. Realizada novamente orientação dietética e quanto à atividade física.

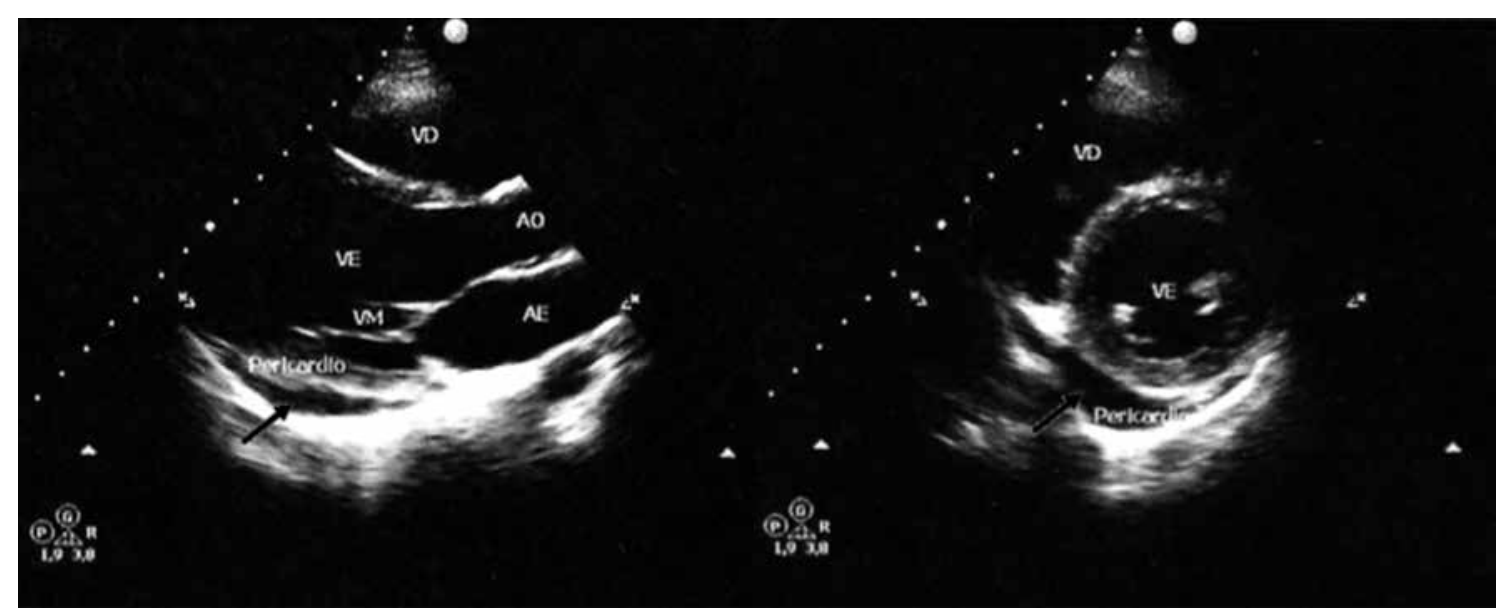

Figura 2. Ecocardiograma de 3/12/2010 evidenciando diminuição significativa do derrame pericárdico (setas). 
Em 16/9/2011, exatamente 1 ano após o episódio inicial, queixava-se de recidiva da astenia e da dor em membros inferiores, além de ganho de $2,5 \mathrm{~kg}$ em três meses, estando com peso de $69 \mathrm{~kg}$. Exames: hemácias $4,31 \times 10^{6} / \mathrm{mL}(4,0-5,2)$, hematócrito $36 \%(37,0$ $48,0)$, hemoglobina $11,9 \mathrm{~g} / \mathrm{dL}(12,0-15,0)$, leucócitos $7.000 / \mathrm{mL}(4.000-10.000)$ e plaquetas $294 \times 10^{3} / \mathrm{mL}$ (150-400); glicemia $83 \mathrm{mg} / \mathrm{dL}$ (70-99); TSH 8,88 $\mathrm{mUI} / \mathrm{L}(0,4-4,0)$ e T4 livre $0,82 \mathrm{ng} / \mathrm{dL}(0,7-1,8)$; colesterol total $267 \mathrm{mg} / \mathrm{dL}(<200)$, HDL colesterol 82 $\mathrm{mg} / \mathrm{dL}(>40)$, LDL colesterol $144 \mathrm{mg} / \mathrm{dL}(<\mathrm{l30})$ e triglicérides $204 \mathrm{mg} / \mathrm{dL}(<150)$. Novo ecocardiograma mostrou derrame pericárdico discreto. Em decorrência do aumento do TSH, houve necessidade de otimizar a dose de levotiroxina para $150 \mathrm{mcg} /$ dia. Ainda, tendo em vista a persistência da dislipidemia apesar das orientações dietéticas e comportamentais, foi iniciado tratamento com rosuvastatina $10 \mathrm{mg}$ ao dia.

\section{DISCUSSÃO}

No passado, o derrame pericárdico representava uma complicação cardiovascular relativamente comum do hipotireoidismo, no entanto, atualmente é uma entidade pouco frequente e que raramente causa sintomatologia (9-11). Por outro lado, o derrame pericárdico maciço, levando a um quadro clínico importante, como o descrito no presente caso, é uma condição incomum, sobretudo sendo o derrame pericárdico a primeira manifestação do hipotireoidismo $(12,13)$.

Destaca-se a forma como o diagnóstico foi feito, em que exames laboratoriais de rotina revelaram um TSH elevado, confirmando o diagnóstico de hipotireoidismo. Esse achado, associado ao do ecocardiograma que demonstrou derrame pericárdico maciço, permitiu estabelecer a associação entre esses dois eventos $(14,15)$ e, por meio disso, a instituição imediata de terapêutica com levotiroxina com pronta melhora clínica da paciente.

Embora o ecocardiograma inicial não tenha evidenciado prejuízo na função cardíaca, a paciente apresentou dispneia importante e edema de membros inferiores com semiologia tipicamente cardiogênica, denotando dissociação clínico-imaginológica (16). Além disso, o ECG demonstrou complexos de baixa voltagem, achado frequente em casos de derrame pericárdico $(1,17,18)$.

A função renal da paciente revelava ureia normal e creatinina aumentada, um padrão compatível com insuficiência renal crônica. No entanto, tal diagnóstico foi afastado após dois exames normais verificados nas consultas subsequentes.

A pesquisa de doenças autoimunes diferentes da tireoidite de Hashimoto, como lúpus eritematoso sistêmico e artrite reumatoide, foi negativa, assim como a avaliação geral para autoimunidade, evidenciada pelo fator antinuclear e pela PCR negativos.

$\mathrm{O}$ seguimento da paciente por $\mathrm{l}$ ano revelou remissão da dispneia, com apenas um episódio desta ao longo do tratamento, além de esvaecimento do edema. No entanto, após a incomum abertura do hipotireoidismo com o derrame pericárdico, mesmo em vigência do tratamento, a paciente veio a apresentar o quadro clínico típico da disfunção tireoidiana: astenia, queda de cabelos, dislipidemia, ganho de peso, irregularidade menstrual, constipação intestinal, anemia, entre outros. Com o tratamento, houve melhora de diversos sintomas, apesar de ter permanecido com astenia e dislipidemia mista com necessidade de estatina, além de ganho de peso de difícil controle ao longo de todo o tratamento $(1,17)$. O perfil hematimétrico melhorou gradativamente com os ajustes no tratamento do hipotireoidismo, com discreta piora na última dosagem hormonal na qual o TSH sofreu pequeno acréscimo. O derrame pericárdico apresentou diminuição volumétrica paulatina, no entanto, sem desaparecer completamente.

A redução do derrame pericárdico ao longo do tempo converge com a otimização da terapêutica com levotiroxina, no entanto, sua remissão é insidiosa, de modo que poderá estar presente por vários meses, ou até anos, mesmo após a instituição da terapêutica $(3,11,15,19,20)$.

Enfatiza-se com este caso a importância de considerar o hipotireoidismo como causa de derrame pericárdico, solicitando precocemente screening tireoidiano em pacientes com este, sobretudo quando se trata de indivíduos jovens, em que a probabilidade de outras etiologias torna-se menor (16,21). Ressalta-se, ainda, a necessidade de considerar que o derrame pericárdico possa ser a primeira manifestação do hipotireoidismo.

Por fim, a evolução do derrame pericárdico para tamponamento cardíaco é rara no hipotireoidismo. Dessa forma, é importante sempre ter em mente que medidas agressivas para o derrame, como a pericardiocentese, janela pericárdica, entre outras, são ineficazes nesses casos, além de potencialmente prejudiciais aos pacientes, e que a melhor conduta é simplesmente a instituição de terapêutica com levotiroxina com o intuito de restabelecer o eutireoidismo (2-4).

Agradecimentos: não houve apoio financeiro ao presente trabalho. 
Declaração: os autores declaram não haver conflitos de interesse científico neste estudo.

\section{REFERÊNCIAS}

1. Silva Jr LFRF, Provenzano SSN, Abreu LM. As alterações cardiovasculares nas doenças tireoidianas. Rev Socerj. 2002;15(1): 21-33.

2. Rachid A, Caum LC, Trentini AP, Fischer CA, Antonelli DAJ, Hagemann RP. Pericardial effusion with cardiac tamponade as a form of presentation of primary hypothyroidism. Arq Bras Cardiol. 2002;78(44):583-5.

3. Lin C, Liu C, Lin T, Chen C, Chen B, Lin C. Myxedema associated with cardiac tamponade. Jpn Heart J. 2003;44(3):447-50.

4. Okumura AC, Uchida M. Tamponamento cardíaco como manifestação de hipotireoidismo. Perspectivas Médicas. 2009;20(1):34-7.

5. Kabadi UM, Kumar SP. Pericardial effusion in primary hypothyroidism. Am Heart J. 1990;120(6 Pt 1):1393-5.

6. Mahroos HA, Bannay RA. Massive pericardial effusion as a sole manifestation of hypothyroidism - A case report. Bahrain Med Bull. 2000;22(4):188-91.

7. Shastry RM, Shastry CC. Primary hipothyroidism with pericardical tamponade. Indian J Pediatr. 2007;74(6):580-1.

8. Baskin HJ, Cobin RH, Duick DS, Gharib H, Guttler RB, Kaplan MM, et al.; American Association of Clinical Endocrinologists. American Association of Clinical Endocrinologists medical guidelines for clinical practice for the evaluation and treatment of hyperthyroidism and hypothyroidism. Endocr Pract. 2002;8(6):457-69.

9. Robillon JF, Sanchez B, Vuolo-Rigaud AM, Creisson G, Gunness S, Morand $\mathrm{P}$, et al. Cardiac tamponade in severe hypothyroidism. A rare cause. Presse Med. 1993;22(26):1221-3.
10. Agenol YE, Esteffan KC, Navea C. Massive pericardial effusion as manifestation of hypothyroidism. Rev Cienc Salud. 2008;12(1):39-42.

11. Omura $Y$, Ugi S, Sugimoto T, Nishio $Y$, Maegawa $H$, Kashiwagi $A$. Massive pericardial effusion secondary to Hashimoto's disease. Eur J Intern Med. 2007;18(5):438-40.

12. Karu AK, Khalife WI, Houser R, VanderWoude J. Impending cardiac tamponade as a primary presentation of hypothyroidism: case report and review of literature. Endocr Pract. 2005;11(4):265-71.

13. Auguet T, Vázquez A, Nolla J, Solsona JF. Cardiac tamponade and hypothyroidism. Intensive Care Med. 1993;19(4):241.

14. Quin JD, McDonald A, Russell R, Thomson JA. Hypothyroidism presenting with cardiac tamponade. Scott Med J. 1994;39(3):82.

15. Hardisty CA, Naik DR, Munro DS. Pericardial effusion in hypothyroidism. Clin Endocrinol (Oxf). 1980;13(4):349-54.

16. Zanettini MT, Zanettini JO, Zanettini JP. Pericardite. Série de 84 casos consecutivos. Arq Bras Cardiol. 2004;82(4):360-4.

17. Fernandes F, Vieira GS, Arteaga E, lanni BM, Fernandes PMP, Mady C. Pericardite por colesterol. Uma causa específica, porém rara de doença pericárdica. Arq Bras Cardiol. 2001;76(5):391-2.

18. DeirosBronte L, Garcia Guereta L, Labrandero de Lera C, Guerrero Fernández J. Hipotiroidismo desenmascarado por derrame pericardico severo. Anpediatr (Barc). 2010;73(1):56-8.

19. Martí CV, Guarinos OJ, Domínguez de Rozas JM. Derrame pericárdico masivo y taponamiento cardíaco como forma de presentación de hipotiroidismo: Reportofone case. Rev Méd Chile. 2005;129(10):1191-4.

20. Hanip MR, Ong SB, Tan TT, Khalid BA. Massive pericardial effusion in primary hypothyroidism. Med J Malaysia. 1989;44(4):341-3.

21. Hsieh CY, Liu BY, Yang YN, Yin WH, Young MS. Massive pericardial effusion with diastolic right ventricular compression secondary to hypothyroidism in a 73-year-old woman. Emerg Med Australas. $2011 ; 23(3): 372-5$. 